\title{
Aspectos anatómicos, clínicos y quirúrgicos de la córnea
} posterior

\section{Anatomical, clinical and surgical aspects of the posterior cornea}

\author{
Carlos E. de la Torre-González ${ }^{1,2 *}$, Ariadna E. García-Castro² y Carmen L. Pérez-Julca ${ }^{2}$
}

${ }^{1}$ Clínica de Córnea, Hospital Juárez de México, Secretaria de Salud; ${ }^{2}$ Curso de alta especialidad en córnea y cirugía refractiva, Universidad Nacional Autónoma de México, Hospital Juárez de México. Ciudad de México, México

\section{Resumen}

El avance en el conocimiento de la ultraestructura de la córnea, que anteriormente podía representar una curiosidad o un dato intelectual para presumir, actualmente es imprescindible para el clínico. Esta revisión tiene por objetivo mencionar los elementos que a juicio de los autores son más relevantes de los aspectos anatómicos (histológicos) «nuevos» de la córnea y como impactan en las también relativamente nuevas técnicas quirúrgicas, enfatizando el papel que tiene la «capa de Dua». La composición del estroma corneal también varía de acuerdo a la profundidad. La obtención del tejido corneal varía de acuerdo a la técnica de disección empleada, si bien es cierto que en la pneumodisección quedan remanentes estromales predesceméticos, estos no constituyen una nueva capa anatómicamente. Las diferencias de hidratación y unión entre las láminas corneales hacen que la córnea se comporte de manera distinta dependiendo del plano de disección. Los cambios que suceden con la edad facilitan la procuración de la membrana de Descemet-endotelio al hacerse más gruesa y fácil de manipular.

Palabras clave: Injerto laminar corneal. DSAEK. DMEK. DALK. Anatomía corneal. Capa de Dua.

\begin{abstract}
The advance in the knowledge of corneal ultrastructure that previously would have been considered a curiosity or just intellectual data, today is essential for the clinician. The purpose of this review is to address the elements that, based on the authors" criteria, are more relevant to the "new" anatomical (histological) aspects of the cornea and how they affect the relatively new surgical techniques, emphasizing the role of "Dua's layer." The composition of the corneal stroma also varies according to depth. Corneal tissue obtainment varies according to the dissection technique used, although it is true that in pneumodissection pre-descemet stromal remnants remain, but they do not constitute a new anatomical layer. The differences in hydration and disposition between the corneal lamellae cause the cornea to behave differently depending on the plane of dissection. Age-related changes facilitate the procurement of the descemet-endothelium membrane, as it becomes thicker and easier to manipulate.
\end{abstract}

Key words: Corneal lamellar graft. DSAEK. DMEK. DALK. Corneal anatomy. Dua's layer.

\section{Correspondencia:}

${ }^{*}$ Carlos E. de la Torre-González

Hospital Español torre antigua de consultorios

Av Ejército Nacional 613 int. 601

Col. Granada, Del. Miguel Hidalgo Fecha de recepción: 15-01-2018

C.P. 11560, Ciudad de México, México

E-mail: delatorre_cornea@yahoo.com.mx

Fecha de aceptación: 18-04-2018

DOI: 10.24875/RMO.M18000042
Disponible en internet: 05-09-2018 Rev Mex Oftalmol. 2018;92(5):227-232 www.rmo.com.mx 0187-4519/@ 2018 Sociedad Mexicana de Oftalmología. Publicado por Permanyer México. Este es un artículo Open Access bajo la licencia CC BY-NC-ND (http://creativecommons.org/licenses/by-nc-nd/4.0/) 


\section{Introducción}

Debido a la gran relevancia que tiene la córnea, no solo como el elemento óptico más importante del ojo sino además por la amplia gama de patologías de las que es susceptible desde el nacimiento hasta la vejez y aún más por la posibilidad de emprender tratamientos en ella de una gran variedad, desde distintos tipos de láser hasta procedimientos químicos como la quelación, o bien su sustitución de forma casi total o en alguna de sus capas mediante los trasplantes penetrantes y laminares, entre otros, el estudio de su fisiología y, en particular, de su anatomía son objeto de gran interés en las áreas clínicas y básicas. El avance en el conocimiento de la ultraestructura de la córnea que anteriormente podía representar una curiosidad o un dato intelectual para presumir, actualmente es imprescindible para el clínico. Esta revisión tiene por objetivo mencionar los elementos que a juicio de los autores son más relevantes de los aspectos anatómicos (histológicos) «nuevos» de la córnea y como impactan en las también relativamente nuevas técnicas quirúrgicas, enfatizando el papel que tiene la «capa de Dua» y la controversia que llegó a ocasionar en distintos foros nacionales e internacionales, además de correlacionar de forma visual con fotografías clínicas, de gabinete y de histología diversos casos de la práctica.

\section{Anatomía e histología corneal aplicadas a la clínica}

La córnea consta de cinco capas: epitelio, capa de Bowman, estroma, membrana de Descemet y endotelio. No es objeto de este trabajo abordar los aspectos de la córnea anterior (epitelio y capa de Bowman), aunque su relevancia no debe ser soslayada debido al alto impacto que tiene la interfaz aire-película lagrimal en el sistema óptico.

Como sabemos, la transparencia de la córnea depende del grado de regularidad espacial en la disposición de las fibras, que conforman láminas que discurren de manera organizada ortogonalmente y que constituyen el "esqueleto» de la córnea, además de ser una estructura delgada y los efectos de interferencia destructiva entre las ondas de luz dispersadas. En el centro de la córnea, las láminas son más escasas, alrededor de 300, mientras que en la periferia aumentan en la cercanía del limbo hasta aproximadamente 500. Las lamelas en la parte posterior de la córnea son continuas, de limbo a limbo, hasta la unión corneoescleral, con una disposición circular en ese sitio, formando lo que Radner ${ }^{1}$, mediante microscopía de barrido electrónico, denominó el «ligamento circular corneal». Las lamelas en la porción posterior de la córnea se encuentran más organizadas, con mayor hidratación ${ }^{2}$, un menor índice refractivo con respecto a la córnea anterior, además su disposición es paralela a la curvatura corneal posterior y son más gruesas y anchas (100 a $200 \mu \mathrm{m}$ de largo y 1 a 2.5 de grueso vs. 0.5 y 0.2 a $1.2 \mu \mathrm{m}$, respectivamente). Esta diferencia histológica en organización y grosor permite, en la clrior ya sea con técnicas manualeas como el aire enomina el ligamento circular corneal.de Bowman, estroma, Descemet y endotelio.ínica, que el estroma posterior sea más sencillo de disecar respecto al estroma anterior, ya sea con técnicas manuales o con el uso de diversas sustancias como el aire o solución que encuentran con mayor facilidad un plano de disección. A pesar de esta relativa facilidad, queda claro que no está exenta de la resistencia que le confieren las uniones entre las láminas y las proteínas de la matriz extracelular, como los proteoglicanos ${ }^{3}$. Es importante resaltar que, a diferencia de la organización paralela a la superficie posterior de las láminas en el estroma posterior, las láminas en el estroma anterior son abigarradas, llegan incluso a transcurrir de forma anteroposterior, y atraviesan otras láminas en su trayecto hasta llegar a su destino. Esta disposición en mosaico, en algunos casos, puede apreciarse clínicamente tras la instilación de fluoresceína.

La composición del estroma corneal también varía de acuerdo a la profundidad. Los glucosaminoglicanos de estroma corneal son el queratán sulfato, dermatán sulfato y la condritina. El primero es el más abundante y el que tiene mayor afinidad por el agua, y se encuentra en mayor cantidad en el estroma posterior, por lo que el estroma anterior es más compacto en tanto que la porción posterior es más propensa al edema 4 .

En 2002, Anwar publicó su artículo en el cual describe la forma de «exponer la membrana de Descemet» mediante el uso de la gran burbuja ${ }^{5}$, la cual, según su autor, separaba la membrana de Descemet del estroma más posterior haciendo la descripción de la burbuja tipo 1. Otros autores han reportado técnicas similares, ya sea con aire, viscoelástico o solución salina, para la realización de un trasplante laminar anterior ${ }^{6-8}$. Sin embargo, esto no es completamente cierto y ha dado lugar a confusión, como se verá más adelante.

La revolución de los injertos laminares anteriores y posteriores se debe a la forma más controlada y reproducible de la disección corneal. Sin lugar a duda, 
aunque después de mucho debate y gracias a los metaanálisis que al respecto se han llevado a cabo, las técnicas de trasplantes laminares de injerto corneal son el procedimiento de elección en el tratamiento de la patología corneal anterior y posterior. Las modalidades más comunes son la queratoplastia laminar anterior profunda (DALK) (Fig. 1), para la patología anterior, $y$, para la patología corneal posterior, la queratoplastia endotelial automatizada con retiro de la membrana de Descemet (DSAEK) (Fig. 2) y la queratoplastia endotelial de membrana de Descemet (DMEK) (Fig. 3). En ambas técnicas es importante separar la membrana de Descemet del estroma posterior, tanto en el donador como en el receptor, para posteriormente agregarle la parte sana que haga falta al receptor, y lograr así ventajas que el injerto penetrante no puede superar ${ }^{9-11}$.

En el 2003, Dua, et al. ${ }^{12}$, basándose en su amplia experiencia en la técnica DALK y su gran capacidad de observación, postularon la existencia de una nueva capa denominada "capa de Dua» en su trabajo «Redefiniendo la anatomía corneal humana» al percatarse que siguiendo la técnica de la gran burbuja y obtener una burbuja tipo 1 se logra un plano de clivaje en la porción preeescemética del estroma, que mide en promedio $10.15 \pm 3.6 \mu \mathrm{m}$ antes de llegar a la membrana de Descemet. Hacen énfasis que la disección no ocurre realmente entre la membrana de Descemet y el estroma, como se había pensado anteriormente, y es posible incluso retirar la membrana de Descemet sin romper la gran burbuja de aire contenida en este espacio en el estroma más profundo. Dichos hallazgos son indiscutibles y replicables, como se aprecia en la figura 4, en botones esclerocorneales no aptos para uso clínico tomados y disecados por los autores de esta revisión. Queda claro, entonces, que existe un plano de clivaje en el estroma profundo que también es visible por tomografía de coherencia óptica (Fig. 5), aunque realmente esto no puede ser considerado como una nueva capa ni redefine nada en la anatomía corneal, como se comenta en el editorial publicado en la misma revista poco tiempo después ${ }^{13}$.

Con la finalidad de corroborar los hallazgos de Dua se realizó un estudio multicéntrico para redefinir la estructura del estroma posterior ${ }^{14}$. En la microscopia de luz, como era esperado, no se encontró ninguna línea de demarcación en el estroma posterior y se observaron queratocitos a diferentes distancias de la membrana de Descemet, a diferencia de lo postulado de una capa de Dua acelular. La microscopia de transmisión confirmó la distancia variable entre la Descemet y los queratocitos, y se encontraron tan cerca como $1.5 \mu \mathrm{m}$

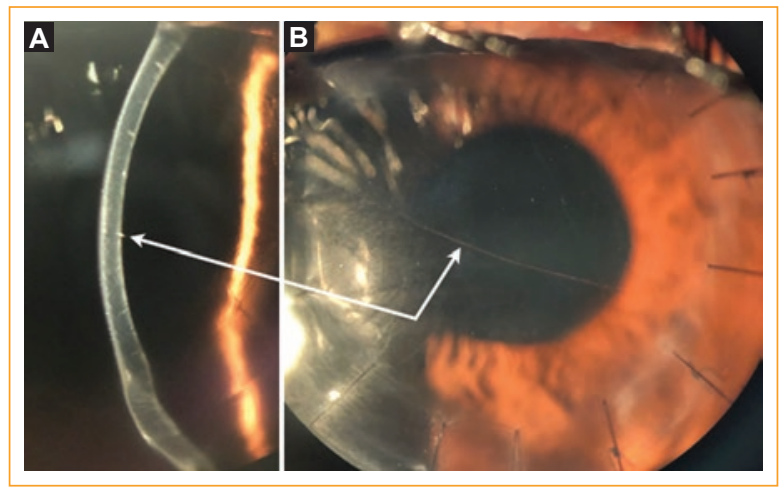

Figura 1. A: Corte de luz en cirugía de queratoplastia laminar anterior profunda sin evidencia de interfaz. B: lluminación directa donde se aprecia pliegue de la membrana de Descemet del receptor.

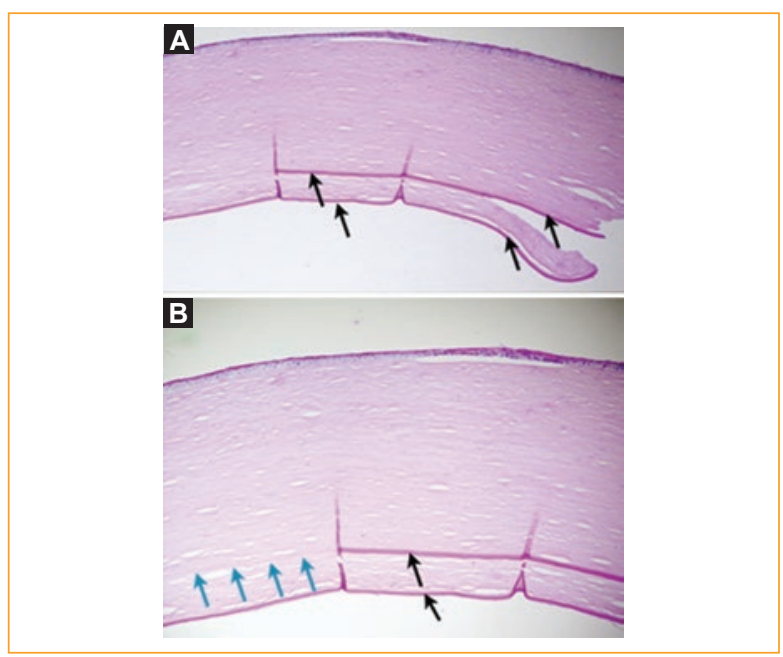

Figura 2. A: Fotomicrografía a gran aumento que muestra lamelas del estroma profundo y membrana de Descemet, doble membrana de Descemet en la periferia (flechas negras) (Ácido peryódico de Schiff [PAS], amplificación original 40x). B: Se aprecia una línea de interfaz entre el estroma receptor y donador (flechas azules).

en el centro de la córnea, lo cual refuta la existencia de una capa posterior de estroma acelular. En la unión estroma-membrana de Descemet se encontró una capa intermedia de 0.5 a $1 \mu \mathrm{m}$ formada por colágeno de disposición irregular, con fibras de diámetro de menor tamaño (21.5 a $2.1 \mathrm{~nm}$ ), que no estaban organizadas en haces semejante a la capa de Bowman, que es fuertemente positiva para colágeno tipo III por inmunofluorescencia.

Si bien es cierto que existen diferentes modalidades de procuración del donador ${ }^{15}$, ya sea con técnicas 


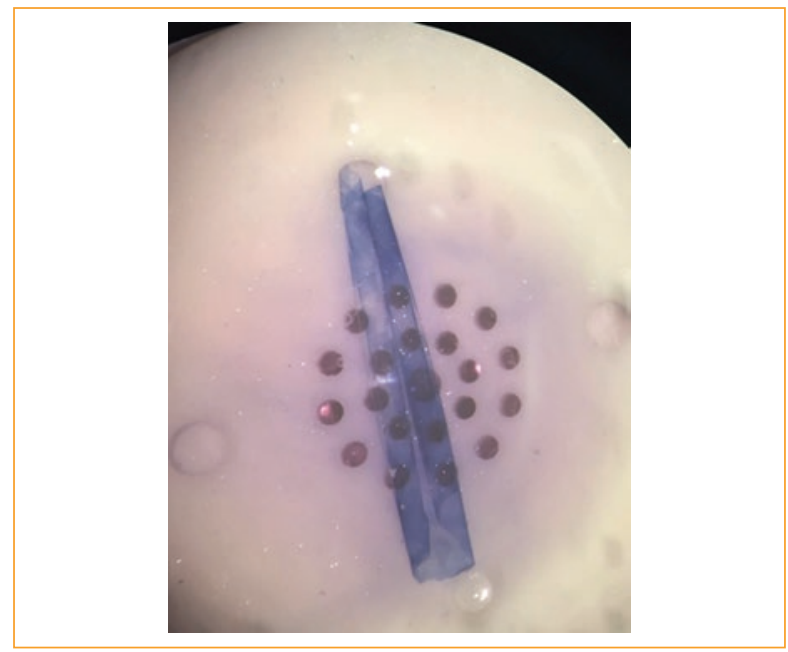

Figura 3. Formación de doble rollo (pergamino) de membrana de Descemet con endotelio en parte externa para cirugía de queratoplastia endotelial de membrana de Descemet (tinción con azul tripano).

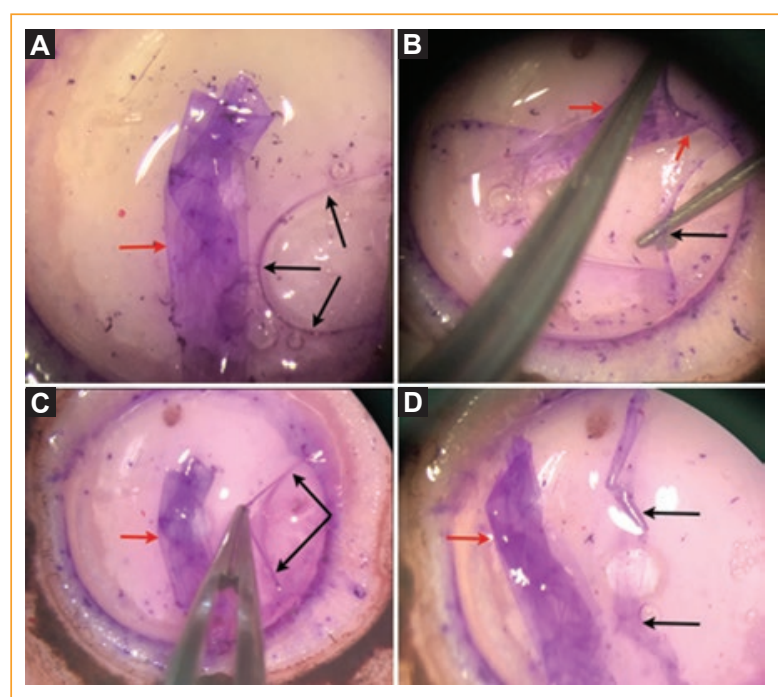

Figura 4. A: descematorrexis de donador, teñido con azul tripano (flecha roja), se aprecia burbuja de aire en el estroma profundo (flechas negras). Endotelio y Descemet (flechas rojas) con cánula (B) y pinza (C) en el espacio formado en el estroma posterior, evidenciando la llamada "capa de Dua». D: disección de endotelio y Descemet (flecha roja) y estroma profundo predescemético "capa de Dua».

manuales ${ }^{16}$, de pneumo o viscodisección (Fig. 6), para la técnica DMEK la pérdida endotelial observada posterior a la preparación del donador es del 2 al $8 \% \%^{17,18}$. Mientras que en los reportes a 6 meses del injerto, la pérdida (cuando se reporta) es alta y va desde el 19 hasta el $44 \%{ }^{19,20}$, dependiendo del autor revisado,

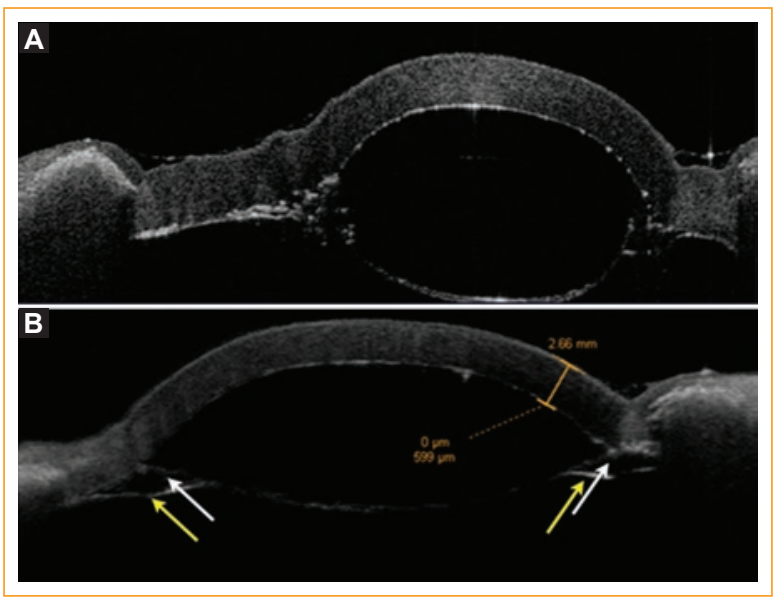

Figura 5. Tomografía de coherencia óptica A: burbuja tipo 1 pequeña, bien delimitada, con pneumodisección de estroma y Descemet. B: Pneumodisección, se aprecia separación del endotelio y Descemet (flecha amarilla) del estroma predescemético (flecha blanca).

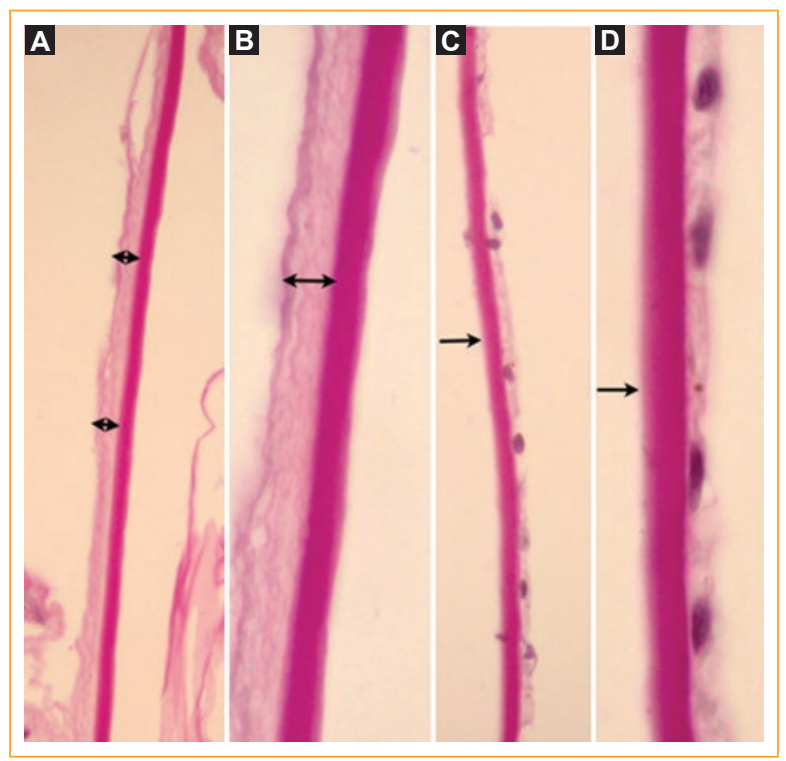

Figura 6. A y B: fotomicrografía a gran aumento de tejido corneal obtenido por pneumodisección que muestra lamelas del estroma profundo y membrana de Descemet, sin presencia de células endoteliales (Ácido peryódico de Schiff (PAS), amplificación original 40x y 100x). C y D: tejido obtenido sin pneumodisección con técnica manual Scuba en la que se aprecia únicamente membrana de Descemet y endotelio sin la presencia de láminas de colágeno del estroma predescemético (PAS, amplificación original 40x y 100x).

tendiendo a estabilizarse después del primer año. Es importante hacer mención de que, aunque teóricamente el anhelo de realizar dos procedimientos de donación en receptores a partir de una sola córnea para 
paliar la escasez de tejido donador, este pudiera resultar contraproducente al menos en sitios donde la procuración de Descemet no es muy común e, incluso, en lugares habituados a estos procedimientos en los que se llega a reportar la pérdida del tejido durante su manipulación en casi una cuarta parte de los $\operatorname{casos}^{17,21}$. De las técnicas empleadas parce ser que la disección manual con técnica Scuba es más reproducible y permite la obtención de áreas más grandes de la membrana de Descemet. Una estrategia que disminuye esta pérdida durante la preparación y facilita la disección del tejido es el utilizar córneas de donadores añosos $^{22}$, cuyas implicaciones clínicas no son el objeto de esta revisión ${ }^{23-25}$.

La córnea posterior presenta cambios con la edad, no únicamente por la pérdida endotelial fisiológica que se calcula en $0.6 \%$ anual ${ }^{26}$, sino además por los cambios que ocurren en la membrana de Descemet, cuya descripción data de 1758. Las primeras lamelas de colágeno de la membrana de Descemet son secretadas en el útero por las células endoteliales a los 4 meses de gestación, que se condensan formando una banda a los 8 meses de edad gestacional y al nacimiento es esta porción anterior la única presente de la membrana de Descemet, con un grosor de $3 \mu \mathrm{m}$. Después del nacimiento se sigue produciendo una segunda porción posterior de la membrana, la cual es homogénea, a diferencia de la anterior, y va engrosándose con la edad, aunque de forma muy variable entre cada individuo, igual que sucede con la pérdida endotelial fisiológica, por lo que este parámetro no podría ser de utilidad para la estimación de la edad de una persona, si bien como mayor sea el individuo esta será más gruesa y el conteo endotelial será también menor $^{27}$. Este aumento del grosor de la capa de Descemet con la edad permite que sea más sencilla de manipular en donadores añosos y en algunas otras condiciones de engrosamiento de la misma, como se aprecia en la descematorrexis de pacientes con distrofia de Fuchs, en quienes es muy sencillo realizarla.

\section{Conclusiones}

La obtención del tejido corneal varía de acuerdo a la técnica de disección empleada, si bien es cierto que en la pneumodisección quedan remanentes estromales predesceméticos, estos no constituyen una nueva capa anatómicamente. Las diferencias de hidratación y unión entre las láminas corneales hacen que la córnea se comporte de manera distinta dependiendo del plano de disección. Los cambios que suceden con la edad facilitan la procuración de la membrana de Descemet-endotelio al hacerse más gruesa y fácil de manipular. Es importante que el cirujano de córnea tenga en cuenta estas consideraciones anatómicas para entender el comportamiento del tejido en su práctica quirúrgica.

\section{Agradecimientos}

Los autores desean agradecer la colaboración del Departamento de Patología de la Asociación para evitar la ceguera en México y en particular al Dr. Abelardo Rodríguez Reyes por su valiosa ayuda en el procesamiento y análisis histológico.

\section{Bibliografía}

1. Radner W, Zehetmayer M, Aufreiter R, Mallinger R. Interlacing and cross-angle distribution of collagen lamellae in the human cornea. Cornea. 1998;17(5):537-43.

2. Freund DE, McCally RL, Farrell RA, Cristol SM, L'Hernault NL, Edelhauser HF. Ultrastructure in anterior and posterior stroma of perfused human and rabbit corneas. Relation to transparency. Invest Ophthalmol Vis Sci. 1995;36(8):1508-23

3. Meek KM, Blamires T, Elliott GF, Gyi TJ, Nave C. The organisation of collagen fibrils in the human corneal stroma: a synchrotron X-ray diffraction study. Curr Eye Res. 1987;6(7):841-6.

4. Bettelheim FA, Plessy B. The hydration of proteoglycans of bovine cornea. Biochim Biophys Acta. 1975;381(1):203-14.

5. Anwar M, Teichmann KD. Big-bubble technique to bare Descemet's membrane in anterior lamellar keratoplasty. J Cataract Refract Surg. 2002;28(3):398-403.

6. Archila EA. Deep lamellar keratoplasty dissection of host tissue with intrastromal air injection. Cornea. 1984;3(3):217-8

7. Loewenstein A, Lazar M. Deep lamellar keratoplasty with complete removal of pathological stroma for vision improvement. Br J Ophthalmol. 1998;82(2):205

8. Sugita J, Kondo J. Deep lamellar keratoplasty with complete removal of pathological stroma for vision improvement. $\mathrm{Br} J$ Ophthalmol. 1997:81(3):184-8.

9. Shimazaki J, Ishii N, Shinzawa M, Yamaguchi T, Shimazaki-Den S, Satake Y. How Much Progress Has Been Made in Corneal Transplantation? Cornea. 2015;34 Suppl 11:S105-11.

10. Nanavaty MA, Wang X, Shortt AJ. Endothelial keratoplasty versus penetrating keratoplasty for Fuchs endothelial dystrophy. Cochrane Database Syst Rev. 2014(2):CD008420.

11. Keane M, Coster D, Ziaei M, Williams K. Deep anterior lamellar keratoplasty versus penetrating keratoplasty for treating keratoconus. Cochrane Database Syst Rev. 2014(7):CD009700.

12. Dua HS, Faraj LA, Said DG, Gray $T$, Lowe J. Human corneal anatomy redefined: a novel pre-Descemet's layer (Dua's layer). Ophthalmology. 2013;120(9):1778-85.

13. McKee HD, Irion LC, Carley FM, Brahma AK, Jafarinasab MR, Rahmati-Kamel M, et al. Re: Dua et al.: Human corneal anatomy redefined: a novel pre-Descemet layer (Dua's layer) (Ophthalmology 2013;120:177885). Ophthalmology. 2014;121(5):e24-5.

14. Schlotzer-Schrehardt U, Bachmann BO, Tourtas T, Torricelli AA, Singh A Gonzalez S, et al. Ultrastructure of the posterior corneal stroma. Ophthalmology. 2015;122(4):693-9.

15. Birbal RS, Sikder S, Lie JT, Groeneveld-van Beek EA, Oellerich S, MeIles GRJ. Donor Tissue Preparation for Descemet Membrane Endothelial Keratoplasty: An Updated Review. Cornea. 2018;37(1):128-35.

16. Melles GR, Lander F, Rietveld FJ. Transplantation of Descemet's membrane carrying viable endothelium through a small scleral incision. Cornea. 2002;21(4):415-8.

17. Krabcova I, Studeny P, Jirsova K. Endothelial quality of pre-cut posterior corneal lamellae for Descemet membrane endothelial keratoplasty with a stromal rim (DMEK-S): two-year outcome of manual preparation in an ocular tissue bank. Cell Tissue Bank. 2013;14(2):325-31. 
Rev Mex Oftalmol. 2018;92

18. Ignacio TS, Nguyen TT, Sarayba MA, Sweet PM, Piovanetti O, Chuck RS et al. A technique to harvest Descemet's membrane with viable endothelial cells for selective transplantation. Am J Ophthalmol. 2005;139(2):325-30.

19. Gorovoy IR, Gorovoy MS. Descemet membrane endothelial keratoplasty postoperative year 1 endothelial cell counts. Am J Ophthalmol. 2015;159(3):597-600 e2

20. Studeny P, Farkas A, Vokrojova M, Liskova P, Jirsova K. Desceme membrane endothelial keratoplasty with a stromal rim (DMEK-S). $\mathrm{Br}$ Ophthalmol. 2010;94(7):909-14.

21. Tausif HN, Johnson L, Titus M, Mavin K, Chandrasekaran N, Woodward MA, et al. Corneal donor tissue preparation for Descemet's membrane endothelial keratoplasty. J Vis Exp. 2014(91):51919.

22. Heinzelmann S, Huther S, Bohringer D, Eberwein P, Reinhard T, Maier P. Influence of donor characteristics on descemet membrane endothelia keratoplasty. Cornea. 2014;33(6):644-8.
23. Venzano D, Pagani P, Randazzo N, Cabiddu F, Traverso CE. Descemet membrane air-bubble separation in donor corneas. J Cataract Refract Surg. 2010;36(12):2022-7.

24. Zarei-Ghanavati S, Zarei-Ghanavati M, Ramirez-Miranda A Air-assisted donor preparation for DMEK. J Cataract Refract Surg. 2011;37(7):1372; author reply

25. Zarei-Ghanavati S, Khakshoor H, Zarei-Ghanavati M. Reverse big bubble: a new technique for preparing donor tissue of Descemet membrane endothelial keratoplasty. Br J Ophthalmol. 2010;94(8):1110-1.

26. Bourne WM, Nelson LR, Hodge DO. Central corneal endothelial cell changes over a ten-year period. Invest Ophthalmol Vis Sci. 1997;38(3):779-82.

27. Johnson DH, Bourne WM, Campbell RJ. The ultrastructure of Descemet's membrane. II. Aphakic bullous keratopathy. Arch Ophthalmol. 1982; 100(12):1948-51. 